\title{
ANÁLISIS
}

\section{ADAPTACIÓN DE LOS MEDIOS TRADICIONALES A LA INNOVACIÓN DE LOS METAMEDIOS}

\section{Adaptation of traditional media to the metamedia innovation}

\section{Francisco Campos-Freire}

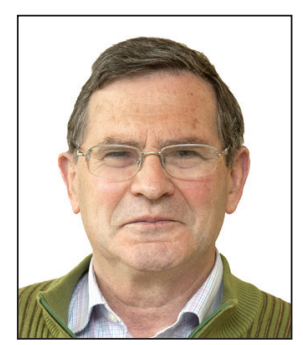

Francisco Campos-Freire es doctor por la Universidad de Santiago de Compostela (USC) y profesor de su Facultad de Ciencias de la Comunicación desde 1991. Es miembro del Grupo de Novos Medios de la USC, coordinador de la Red Internacional de Investigación de Gestión de los Medios de Comunicación (R2014/026 Xescom) e investigador del Programa Prometeo del Senescyt en las Universidades Técnica Particular de Loja y Pontificia Católica de Ibarra en Ecuador. http://orcid.org/0000-0003-1831-1838

Universidad de Santiago de Compostela, Facultad de Ciencias de Comunicación Av. Castelao, s/n. 15705 Santiago, España francisco.campos@usc.es

\section{Resumen}

El objetivo de este trabajo es observar cómo la innovación disruptiva generada por las tecnologías, procesos y sistemas de gestión de la información (creación, recuperación, circulación y acceso), que se materializa a través de los metamedios sociales surgidos de la Web, es adoptada por los medios de comunicación tradicionales para reequilibrar sus modelos de negocio y redes de valor. La prensa impresa europea ha perdido en los últimos años cerca de 50 millones de ejemplares diarios de difusión y la televisión está viendo cómo se fragmentan sus audiencias y disgregan sus inversiones publicitarias. Muchos diarios dejaron de imprimirse en todo el mundo y otras grandes organizaciones de medios han cambiado de manos o se han sometido a fuertes reestructuraciones. El crecimiento mundial previsible de la industria mediática hasta 2018 es del 5,5\% anual pero el de la publicidad digital, en cambio, se estima en más del 10,7\% cada año.

\section{Palabras clave}

Medios; Medios digitales; Metamedios; Transmedia; Hipermedia; Innovación disruptiva; Modelo de negocio; Red de valor; Redes sociales.

\begin{abstract}
The aim of this study is to observe how the disruptive innovation generated by technologies, processes and information management systems (creation, recovery, traffic, and access), which is implemented through social metamedia on the Web, are adopted by traditional media to rebalance their business models and value-added networks. In the last ten years, print media in Europe have lost more than 50 million copies in daily circulation, and television is suffering from audience fragmentation and loss of advertising sales. Many newspapers around the world have stopped printing and other large media companies have changed hands or undergone major restructuring. The global media industry's annual growth is expected to be $5.5 \%$ until 2018 but digital advertising is estimated at $10.7 \%$ per year.
\end{abstract}

\section{Keywords}

Media; Online media; Metamedia; Transmedia; Hypermedia; Groundbreaking innovation; Business models; Value networks; Social networks.

Campos-Freire, Francisco (2015). "Adaptación de los medios tradicionales a la innovación de los metamedios". El profesional de la información, v. 24, n. 4, pp. 441-450. 


\section{Introducción, método y teoría}

Este artículo trata de explicar, a partir de las teorías de la innovación disruptiva y de la rearticulación de las redes de valor de las organizaciones, los cambios que están experimentando los modelos de negocio de los medios tradicionales y los nuevos medios digitales (metamedios, según el concepto de Manovich, 2008). Se realiza, en primer lugar, una aproximación teórica a los conceptos y fenómenos que gravitan sobre el ecosistema mediático actual, para presentar a continuación los principales datos, resultados y tendencias que forman parte de la agenda de investigación y gestión de la industria de la comunicación.

El trabajo se apoya en metodología cuantitativa y cualitativa para el contraste de datos del Sistema de Análisis de Balances Ibéricos (SABI, 2014), de la Asociación Mundial de Diarios (WAN-IFRA, 2014), del Observatorio Audiovisual Europeo (OBS, 2013) y de la evaluación de tendencias de otras fuentes empresariales (PWC, 2014). La hipótesis operativa es que los procesos tecnológicos y organizativos disruptivos de los nuevos medios o metamedios acaban siendo adoptados por los medios tradicionales para recomponer sus mercados y modelos de negocio.

Las innovaciones disruptivas alteran las estructuras de mercado, las redes de valor y los modelos de negocio

El estudio de los nuevos medios de comunicación es una prioridad que Albarran (2014) sitúa entre las líneas a desarrollar por la investigación de la gestión y que aquí se contextualiza, partiendo del concepto de metamedio social, como resultado de la innovación disruptiva. Es un objeto propio de la gestión mediática, entendida tanto desde la administración de los medios como del análisis de sus resultados económicos o de la misma economía política (Albarran, 1997; Sinclair, 2000; Picard, 2006). Un campo de estudio complejo e híbrido, que se complica por los acelerados cambios (Fine, 2000; Küng, 2008) que protagoniza la industria mediática.

La revolución tecnológica provocada por la introducción de la computadora y el desarrollo del software, encarnada en la representación de los nuevos metamedios (Manovich, 2008; 2013), es una innovación discontinua y disruptiva a la vez. Discontinua porque en sus primeros aspectos o fases (Web 1.0) no afectaba radicalmente -suponía un cambio, pero sólo incremental, de evolución y mejora- con respecto a los ámbitos mediáticos tradicionales y disruptiva porque en sus desarrollos o aplicaciones posteriores (web social 2.0 y semántica 3.0) acaba generando nuevos mercados y redes de valor diferentes.

La teoría de la innovación, que se remonta al análisis económico de Schumpeter (1943), presupone un proceso de nuevo emprendimiento, a partir de creaciones e invenciones que provocan movimientos dinámicos de "destrucción creativa", donde las empresas más activas desplazan a las que se han quedado rezagadas en el desarrollo de sus productos, procesos, comercialización y/o organización. Según la OCDE (2010), la innovación es la introducción de un producto (bien o servicio) o de un proceso, nuevo o significativamente mejorado, mediante la aplicación de un renovado método de comercialización o de organización de las prácticas de negocio así como de las relaciones laborales internas y sociales externas. La innovación puede ser continua, adaptativa, disruptiva y transformadora. La primera y la segunda corresponden a las industrias tradicionales mientras que la tercera y cuarta son las que cambian las reglas de juego y crean nuevos mercados.

La innovación disruptiva, según Clayton M. Christensen y Joseph Bower $(1995,1997)$, se genera a través de una destrucción creativa capaz de formar nuevos mercados, modelos de negocio y redes de valor que trastocan y acaban perturbando los ámbitos económico-comerciales tradicionales. El dilema innovador de Christensen (1997, 2014), a diferencia del enfoque económico schumpeteriano, enfatiza el rol disruptivo económico-comercial de las tecnologías e innovaciones en torno a tres elementos críticos:

- distinción entre innovación sostenida y disruptiva,

- tasa de mejoras que los usuarios pueden absorber, y

- evolución del mercado a medida que se introducen nuevos productos o servicios.

Las innovaciones disruptivas alteran las estructuras de mercado (Küng, 2008) y, por lo tanto, los modelos de negocio y sus redes de valor. Crean nuevos mercados y relaciones que los operadores tradicionales no saben o no pueden aprovechar por incapacidad de adaptación: baja rentabilidad, dificultad de monetización e insostenibilidad de sus pesadas o rígidas estructuras.

La innovación sostenida es la mejora que introducen las empresas tradicionales con el objetivo de evolucionar en sus propios mercados, desde la aplicación del color en la emisión de televisión o en la impresión de la prensa, a partir de la década de los años 80 del pasado siglo XX. Luego también la creación de ediciones digitales simultáneas mediante la Web 1.0. O de igual manera en la comercialización de la prensa mediante la entrega gratuita o promoción a través de otros productos de valor añadido (culturales o de consumo), a finales de la década de los años 90, así como en la radiodifusión a través de tecnología digital, en la primera década del siglo XXI. Y lo es ahora -en respuesta a la crisis de inversión comercial- la denominada publicidad nativa, de publirreportaje o emplazamiento inmersivo dentro del contenido (product placement), que reporta más altos ingresos pero también una fisura en el debate ético sobre la credibilidad, la promiscuidad y la frontera que separa el periodismo de la publicidad (Sullivan, 2013).

La innovación disruptiva es la que imponen los nuevos competidores entrantes (Porter, 2009) de una industria, los nativos digitales, con mejoras tecnológicas diferenciadoras, pero más bajas prestaciones (lower-end), para un consumidor con menos poder adquisitivo y exigencia, pero que acaba engrosando un nuevo mercado al que difícilmente pueden acceder las empresas establecidas, por su baja rentabilidad. Este cambio discontinuo acaba imponiendo la necesidad de adaptación de los mercados y las organizaciones. Este es el dilema disruptivo de las empresas de medios 
tradicionales. No es que estos últimos no sean innovadores -que sí lo son en muchos casos- sino que les cuesta mucho más adaptarse a la innovación disruptiva porque sus estructuras de negocio y valor no son adecuadas para el cambio discontinuo (Küng, 2008; Christensen, 2014).

La ventaja competitiva del dilema innovador de Christensen (1997) se amplía actualmente a través de nuevos factores disruptivos (Shaughnessy, 2015), tales como tecnologías emergentes (3D, 5G), ubicuidad y capacidad de conexión de la gente a través de la nube, redes globales de interacción, uso del software libre y la descentralización creativa, flexibilidad y desarrollo de plataformas adyacentes de meta servicios, transacciones P2P sin monetización o con nuevas monedas (bitcoin), colaboración a través de redes de micro tareas y negocios laterales o modelos inversos de uso abierto de big data a partir de tecnologías VRM (vendor relationship management) como las promovidas por el Berkman Center for Internet \& Society de la Harvard University. https://cyber.law.harvard.edu

Todo eso es capitalizado principalmente por los medios nativos digitales, lo que les permite desarrollar, a través de ecosistemas globales y flexibles, nuevos modelos de economía, autocomunicación ${ }^{1}$, blogs, redes sociales, Wikipedia, crowdsourcing, crowdfunding, etc.

\section{Los metamedios digitales desarrollan nuevos modelos de economías de la atención, colaboración, participación y likes, nuevos modelos de negocio y capi- tal social virtual}

La innovación tecnológica del software libre y de la Web 2.0 (O’Reilly, 2005) permitió la creación y sostenimiento de nuevos medios de autocomunicación (Castells, 2009) basados en economías de la atención (Davenport; Beck, 2002), de la colaboración (Tapscott; Williams, 2007), de la participación (Jenkins, 2008, 2009 y 2010; Noguera-Vivo, 2014), de los big data (Mayer-Schönberger; Cukier, 2013), de la nube (Schmidt; Cohen, 2014), de los likes (Rushkoff, 2014) y de las redes sociales digitales (Benghozi, 2011). Estos modelos dan lugar al mismo tiempo a nuevas redes de valor -conjunto de roles e interacciones que generan un negocio o bien social (Allee; Schwabe, 2011)- más amplias o diferentes a las que se establecían a través de los medios tradicionales. Las redes sociales digitales son redes de valor cuya representación relacional es el capital social virtual (Bourdieu, 1986; Coleman, 1988; Putnam, 1993; Burt, 1992; Granovet- ter, 1974; Lin, 2001; Requena, 2003; Damásio; Henriques, 2011; y otros).

El concepto de metamedio, de inspiración macluhiana y desarrollo de Kay y Goldberg (1977), fue retomado por Manovich (2008) para describir la innovación computacional a través de su evolución hipermedia (Nelson, 1965; Scolari, 2008) y capacidad transmedia (Kinder, 1991). Los nuevos medios son metamedia y postmedia porque utilizan la materia prima de los medios tradicionales y la evolución de las técnicas informáticas "existentes o aún no inventadas" para reinventarse (Manovich, 2008, p. 92) a través de un continuo proceso de remediación (Bolter; Grusin, 2000) que alcanza a todo el ecosistema mediático. Los ecosistemas y plataformas son contemplados en la teoría de la gestión flexible de las organizaciones, más allá de su tecnología y operatividad, como redes de valor, conexión, relación, colaboración, participación y sociabilidad (Vitalari; Shaughnessy, 2012).

El ecosistema emergente en el que hoy conviven los medios, tradicionales y nuevos, es hipermedia y sus procesos son cada vez más transmedia. La estructura metamedia es la que desarrolla la articulación hipermedia, a través de nodos y enlaces, mediante un sistema narrativo (flujos de información) complejo, conectado, colaborativo, inmersivo, dinámico, abierto, multifactorial y multivariable denominado transmedia (Galán-Ugartemendía, 2012). Esa estructura comunicacional es similar a la de las redes sociales reales, que agrupan a distintos individuos (nodos o actores) que se relacionan entre sí (mediante vínculos o enlaces) con un fin específico, caracterizado por la existencia de flujos de sociabilidad y comunicación.

Danah Boyd y Nicole Ellison $(2007,2013)$ y Thomas Stenger (2009) definen los sitios de redes sociales (social network sites) como plataformas de autocomunicación online en las que los participantes pueden: (1) disponer de perfiles públi- 
cos o semipúblicos asociados a una identificación única que son creados por una combinación de contenidos producidos por los utilizadores, sus amigos y datos sistémicos; (2) exponer públicamente las relaciones susceptibles de ser visualizadas y consultadas por otros a través de una lista de enlaces dentro del sistema; (3) acceder a flujos de contenidos (combinaciones de textos, fotos, vídeos, datos y nuevos enlaces) generados por los usuarios, sus contactos y otros proveedores de contenidos a través de internet; (4) recomendar y convertirse en prescriptores de productos y marcas; (5) formar parte de nuevos modelos de negocio y redes de valor; (6) interconectarse con distintas constelaciones de aplicaciones y dispositivos comunicacionales; (7) intercambiar mensajes, servicios colaborativos y publicidad gestionada por la operadora matriz o asociados; (8) desarrollar conversaciones y transacciones; (9) aplicar dispositivos de inteligencia semántica e interconexiones con Internet de las cosas; (y 10) conformar redes de comunicación, relación y meta servicios digitales que se valorizan en forma de capital social.

\section{Modelos de negocio y redes de valor}

El actual paisaje mediático está dominado por cinco grandes tendencias que Robert G. Picard (2014) identifica como súper abundancia de medios, fragmentación y polarización de audiencias convertidas en prosumidoras, amplio desarrollo del portfolio de ofertas, debilitamiento de las compañías y un poderoso cambio en el sistema de comunicaciones. El deterioro de los mercados tradicionales tiene que ver con esa nueva ecología del ecosistema mediático, con los cambios en las redes de valor de los modelos de negocio y con la pérdida de liderazgo en la innovación por parte de los operadores tradicionales frente "a nuevos actores surgidos en el entorno de las industrias tecno-culturales" (Díaz-Nosty, 2013, p. 152).

El modelo de negocio es la manera como una organización crea, proporciona y captura valor (Osterwalder, 2004; Goyanes-Martínez, 2013) y la red de valor son los roles e interacciones que se establecen a través de la arquitectura y tecnología sistémica (Allee; Schwabe, 2011). El modelo de negocio refleja el valor que una empresa ofrece a sus clientes, a través de su propia estructura y de la del ecosistema de su red de socios, para la creación, comercialización y transacción con el fin de generar nuevos flujos relacionales, así como los ingresos necesarios (Osterwalder; Pigneur; Tucci, 2005).

El modelo de negocio se confunde, a veces, con el de financiación pero es más amplio que éste. Aquel es todo el sistema de articulación comercial (producto, precio, distribución y promoción) y su red de valor transaccional mientras que la financiación y el pago sólo son partes del mismo. Los modelos de negocio tradicionales del sector de la comunicación se desarrollan alrededor de sus variadas propuestas de valor y triples economías mixtas formadas por aportaciones públicas (en algunos casos), pago del consumo del producto o del servicio y publicidad generada por la atención (audiencia), colaboración y participación de los usuarios.

\section{Las redes sociales digitales son un nue- vo paradigma del ecosistema actual de medios}

De carácter general, se pueden identificar las siguientes modalidades de negocio según sus sistemas de transacción (Celaya et al., 2014):

1) De propiedad, financiación y gestión pública (radiotelevisión): Canon, subvención, convenios, préstamos financiados, bonificación y/o exención fiscal.

2) De pago del producto o servicio: Por unidad, paquete, suscripción y alquiler (audiovisual).

3) De publicidad: acceso abierto o libre, patrocinio, bartering, merchandising y product placement (emplazamiento de producto/publicidad nativa).

4) De pago del servicio digital: En audiovisual: triple o múltiple play, pago por visión (PPV), por descarga, por paquete, suscripción y alquiler de streaming. En paywall de prensa: micropagos; venta de artículos sueltos (Blendle Newspaper); muro cerrado de pago total; freemium-premium; pago mediado; y membresía o club. 
5) De agregadores de multiservicios y plataformas de fidelización de clientes (ofrecer contenidos gratis para captar clientes para servicios de pago)

6) De otras formas híbridas y filantrópicas: P2P-mooc's, mecenazgo, crowdfunding, crowdsourcing y gamificación.

\section{Resultados económicos}

La producción audiovisual mundial se acerca a los 400.000 millones de dólares y sigue siendo mayoritariamente norteamericana, con gran crecimiento junto a otros países emergentes, como se aprecia entre 2009 y 2013 (tabla 1). La fuerza de ese crecimiento llega también a Europa donde 35 de los 50 principales grupos audiovisuales de este mercado son norteamericanos, japoneses, coreanos y de otros países emergentes.

Las cifras de evolución del audiovisual europeo revelan su fragmentación, los cambios en el consumo y la rearticulación de nuevas redes de valor. El Observatorio Audiovisual Europeo (OBS, 2013/2014) registra en los 28 países de la Unión Europea 8.828 canales de televisión así como 122.755 empresas audiovisuales, que facturan 130.768 millones y dan empleo a 639.450 personas, con apenas crecimiento en los últimos cinco años. La TV de pago y la venta de equipos digitales para ver el cine en casa marcan tendencia junto al estancamiento de la TV pública y la publicidad tradicional.

\section{Los medios tradicionales viven la transi- ción digital como un complejo proceso de innovación continua y disruptiva}

En el ámbito de la televisión, tomando como referencia el mercado audiovisual europeo (OBS, 2013), hallamos doce modelos: público estatal, público local y regional, comercial generalista estatal, comercial regional y local, temáticos, televenta, redes de distribución, plataformas en abierto y pago, plataformas convergentes con telecomunicaciones y plataformas de operadores de servicios sobre otros servicios ("infomediarios"). Los modelos de canales unitarios de difusión terrestre, satélite y cable evolucionan a plataformas convergentes de oferta multicanal y redes (ecosistemas) de "infomediarios" globales (Netflix, Dailymotion, YouTube, Hulu, Google TV).

La pérdida de difusión de la prensa impresa, de pago y gratuita, es el dato más llamativo de la evolución de los mercados mundiales de medios de comunicación. Entre 2008 y 2013 los mercados europeos de prensa impresa registran una pérdida de difusión de 50 millones de ejemplares diarios, según la Asociación Mundial de Diarios (WAN-IFRA, 2013).

Los datos y porcentajes de la tabla 3 hay que verlos en función de la dificultad de establecer estadísticas homogéneas de todos los países del mundo. La base de datos de WANIFRA no dispone de resultados completos de 2013 aunque sí ofrece porcentajes de evolución indicativos, con respecto a 2009, sobre una muestra amplia de países y continentes. Los porcentajes de 2013 a 2009 son significativos, aunque no representen a la totalidad, porque reflejan un descenso en la prensa impresa del $17,45 \%$ en el mundo y del $23,59 \%$ en Europa. Se mantiene el número de cabeceras, pero con tendencia a la baja.

La inversión publicitaria mundial en medios se acerca al medio billón de dólares $\left(0,5 * 10^{12}\right)$, con un crecimiento medio entre 2009 y 2013 del 15\%, de nuevo con lastre en Europa y más potencialidad en América y Asia. La inversión publicitaria por medios en la Unión Europea (OBS, 2014) descendió entre $2009-2013$ en prensa un $21 \%$, en magazines un $23 \%$

Tabla 1. Producción audiovisual mundial (millones de US\$)

\begin{tabular}{|l|r|r|r|r|r|c|}
\hline Mercados & \multicolumn{1}{c|}{$\mathbf{2 0 0 9}$} & \multicolumn{1}{c|}{$\mathbf{2 0 1 0}$} & \multicolumn{1}{c|}{$\mathbf{2 0 1 1}$} & \multicolumn{1}{c|}{$\mathbf{2 0 1 2}$} & \multicolumn{1}{c|}{$\mathbf{2 0 1 3}$} & \% 2013-2009 \\
\hline EUA & 204.724 & \multicolumn{1}{c|}{220.911} & 232.996 & 244.516 & 264.131 & \multicolumn{1}{c|}{29,01} \\
\hline Europa & 78.324 & 78.024 & 74.261 & 75.677 & 68.500 & $-12,54$ \\
\hline Japón & 60.831 & 60.432 & 53.663 & 48.853 & 49.798 & $-18,13$ \\
\hline otros & 11.057 & 12.616 & 13.599 & 13.976 & 15.112 & \multicolumn{1}{c|}{36,67} \\
\hline Total & 354.936 & 371.983 & 374.519 & 383.022 & 397.541 & 12,00 \\
\hline
\end{tabular}

Elaborado a partir de "2013 Yearbook OBS RTV" (OBS, 2013) 
Tabla 2. Evolución del mercado audiovisual europeo (millones de euros)

\begin{tabular}{|c|c|c|c|c|c|c|c|}
\hline Operadores & 2008 & 2009 & 2010 & 2011 & 2012 & $\begin{array}{c}\% \\
2012-2011 \\
\end{array}$ & $\begin{array}{c}\% \\
2012-2008 \\
\end{array}$ \\
\hline \multicolumn{8}{|c|}{ Televisión en abierto } \\
\hline RTV públicas & 32.370 & 33.434 & 33.815 & 33.687 & 32.626 & -3 & 1 \\
\hline Publicidad TV & 21.664 & 20.096 & 21.139 & 21.941 & 21.057 & -4 & -3 \\
\hline TV temática & 9.021 & 9.791 & 10.859 & 12.334 & 12.578 & 2 & 39 \\
\hline Televenta & 2.442 & 2.423 & 2.577 & 2.719 & 2.800 & 3 & 15 \\
\hline TV local & 1.607 & 1.555 & 1.573 & 1.496 & 1.423 & -5 & -11 \\
\hline Radio privada & 4.167 & 3.893 & 4.040 & 4.168 & 4.115 & -1 & -1 \\
\hline Total TV & 71.270 & 71.192 & 74.002 & 76.345 & 74.599 & -2 & 5 \\
\hline \multicolumn{8}{|c|}{ Ingresos de TV de pago } \\
\hline Cable & 11.201 & 11.314 & 12.050 & 12.328 & 12.952 & 5 & 16 \\
\hline Satélite & 13.255 & 13.298 & 14.954 & 15.470 & 16.219 & 5 & 22 \\
\hline IPTV & 1.395 & 2.042 & 2.899 & 3.343 & 4.168 & 25 & 199 \\
\hline DTT & 598 & 954 & 1.439 & 1.525 & 1.613 & 6 & 170 \\
\hline Total & 26.448 & 27.609 & 31.342 & 32.677 & 34.952 & 7 & 32 \\
\hline \multicolumn{8}{|c|}{ Cine y vídeo } \\
\hline Taquilla cine & 5.626 & 6.087 & 6.373 & 6.445 & 6.570 & 2 & 17 \\
\hline DVD venta & 7.727 & 6.729 & 6.198 & 5.532 & 4.913 & -11 & -36 \\
\hline DVD alquiler & 1.372 & 1.167 & 1.037 & 900 & 763 & -15 & -44 \\
\hline Blu-ray venta & 234 & 500 & 807 & 982 & 1.126 & 15 & 381 \\
\hline Blu-ray alqler & 16 & 42 & 84 & 116 & 163 & 41 & 919 \\
\hline Total vídeo & 9.348 & 8.438 & 8.126 & 7.530 & 6.966 & -7 & -25 \\
\hline \multicolumn{8}{|c|}{ Ingresos de vídeo online } \\
\hline VODTV & 97 & 161 & 298 & 392 & 575 & 47 & 493 \\
\hline VOD cine & 26,8 & 59,0 & 117,2 & 189,2 & 380,9 & 101 & 1321 \\
\hline VOD total & 124 & 220 & 415 & 582 & 596 & 2 & 381 \\
\hline Videojuegos & 10.848 & 10.683 & 11.061 & 11.150 & 10.886 & -2 & 0,35 \\
\hline Total audiovis. & 123.664 & 124.230 & 131.318 & 134.729 & 134.929 & 0,1 & 9 \\
\hline \multicolumn{8}{|c|}{ Empresas audiovisuales europeas, ingresos y empleo } \\
\hline No emprs. AV & & 110.151 & 122.165 & 122.755 & & $0,4^{*}$ & $11^{*}$ \\
\hline Ingresos & & 126.639 & 130.768 & 131.239 & & $0,4^{*}$ & $4^{*}$ \\
\hline Empleo & & 648.053 & 643.603 & 639.450 & & $-1^{*}$ & $-1,3^{*}$ \\
\hline
\end{tabular}

Elaborado con datos del Observatorio Audiovisual Europeo (OBS, 2014). En el apartado de empresas y empleo los datos se refieren sólo a las comparaciones de los años 2011-2010 y 2009, respectivamente. Los porcentajes marcados con asterisco $\left({ }^{*}\right)$ corresponden a la evolución entre los años 2011-2009 y 20112010 respectivamente

y en vallas exteriores en un 0,51\% mientras que subió un $5,12 \%$ en televisión, un $0,41 \%$ en radio, un $6,64 \%$ en cines y un $74,75 \%$ en internet. En redes sociales, los ingresos publicitarios de Facebook, Twitter y Linkedln aumentaron desde los 760 millones de dólares de 2009 a los 9.264 millones de 2014 (Díaz-Nosty, 2013).

Los medios de comunicación tradicionales amplían y readaptan sus modelos de negocio a las redes de valor de los nativos digitales

Si las tendencias de los datos mundiales y europeos de la prensa impresa las ponemos en relación con las cifras de este mismo sector en España, el contraste es aún más alar- mante. La prensa española perdió, entre 2008-2013, un 59\% de su difusión (2,8 millones de ejemplares) y los diarios de pago controlados por OJD disminuyeron sus ingresos en un $34 \%$, al pasar de 3.384 millones a 1.986 millones euros, según datos de WAN-IFRA y SABI. Los beneficios de esos 90 diarios impresos controlados por OJD ascendieron a $198 \mathrm{mi}-$ Ilones en 2013, frente a 146 millones negativos en 2012 y 184 millones positivos de 2008.

La prensa impresa empezó a contraatacar su crisis -que no es coyuntural sino estructural y sistémica, como parte también de la recesión económica general (Campos, 2011)- a través de la reestructuración, innovación y reorganización de sus modelos de negocio. A partir de 2010 cambia su estrategia de ciberdifusión gratuita por la protección de sus derechos digitales (tasa y conflicto con Google News en varios países) e implantación de los primeros muros de pago (paywall) para ayudar a salvar su tradicional sistema 
Tabla 3. Evolución de la difusión de la prensa

\begin{tabular}{|c|c|c|c|c|c|c|c|c|}
\hline \multirow{2}{*}{ Continentes } & 2008 & 2009 & 2010 & 2011 & 2012 & 2013 & $\%$ 2012-2008 & \% 2013-2009 \\
\hline & \multicolumn{7}{|c|}{ Difusión } & \\
\hline África & 9.065 & 9.581 & 10.110 & 12.805 & 5.583 & 1346 & -38 & $-22,34$ \\
\hline Asia & 316.981 & 342.649 & 344.000 & 333.945 & 333.338 & 66.640 & 5 & $-0,04$ \\
\hline América & 81.722 & 78.504 & 76.179 & 75.258 & 72.606 & 15.513 & -11 & $-1,53$ \\
\hline Europa & 129.791 & 117.501 & 105.956 & 99.710 & 91.600 & 78.640 & -29 & $-23,59$ \\
\hline \multirow[t]{2}{*}{ Mundo } & 537.762 & 548.867 & 536.880 & 520.338 & 504.107 & 162.139 & -6 & $-17,45$ \\
\hline & \multicolumn{7}{|c|}{ Número de cabeceras } & \\
\hline África & 178 & 186 & 185 & 185 & 121 & 121 & -47 & 5 \\
\hline Asia & 5.626 & 5.834 & 5.968 & 7.336 & 6.539 & 708 & 14 & 3,82 \\
\hline América & 3.240 & 3.148 & 3.136 & 3.136 & 3.206 & 2.327 & -1 & 6,37 \\
\hline Europa & 2.383 & 2.365 & 2.292 & 2.296 & 2.146 & 1.524 & -11 & $-10,49$ \\
\hline \multirow[t]{2}{*}{ Mundo } & 11.427 & 11.533 & 11.582 & 12.926 & 11.912 & 4.580 & 4 & $-5,13$ \\
\hline & \multicolumn{7}{|c|}{ Inversión publicitaria total en medios } & \\
\hline África & 3.500 & 3.650 & 4.220 & 4.400 & 4.682 & 4.381 & 34 & 20,02 \\
\hline Asia & 113.757 & 114.310 & 127.825 & 135.796 & 140.765 & 137.414 & 24 & 20,21 \\
\hline América & 170.210 & 177.902 & 186.844 & 193.329 & 203.451 & 212.567 & 20 & 19,48 \\
\hline Europa & 115.785 & 116.260 & 122.468 & 126.294 & 122.819 & 121.293 & 6 & 4,32 \\
\hline Mundo & 403.252 & 412.123 & 441.359 & 459.820 & 471.718 & 475.656 & 17 & 15,41 \\
\hline
\end{tabular}

Elaboración con cifras de World Trends WAN-IFRA, 2014. Los datos de difusión son en millones de ejemplares y los de la inversión publicitaria total en medios en millones de dólares. Los datos de 2013 no están completos en la base de WAN-IFRA y por eso la evolución porcentual sólo se refiere a la comparación de los países y años de los que hay cifras homogéneas. En Asia se agregan los datos de Australia, Oceanía, Turquía y Emiratos Árabes.

mixto de ingresos, aunque sin renunciar a captar parte del crecimiento del 10,7\% anual previsto hasta 2018 para la publicidad digital (PWC, 2014). Salvo casos excepcionales (Financial Times, grupo Axel Springer o grandes cabeceras globales), el uso actual del paywall en la prensa digital aún oscila entre el $7 \%$ del Reino Unido y el $14 \%$ de Finlandia, con una media mundial del 10\% (Picard, 2014).

La circulación diaria de la prensa impresa pierde 50 millones de ejemplares en Europa desde 2008

\section{Contraste y conclusión}

La transición digital es un proceso complejo de innovación continua y disruptiva a la vez, que afecta de forma desigual a los distintos sectores, mercados y modelos de negocio mediáticos (WNMN, 2014). Tras una primera etapa de respuesta integradora automática a la convergencia digital, los grandes grupos mediáticos (News Corporation, Gannett, Axel Springer) han reaccionado segregando sus negocios tradicionales de impresión de los digitales y audiovisuales. The New York times (TNYT, 2014) convirtió su estrategia digital en el corazón de la innovación y de la interacción/conversación con sus lectores a través de nuevas redes sociales y de valor. Los medios tradicionales copian la innovación disruptiva de los nativos digitales.

La potencialidad disruptiva de las plataformas, ecosistemas y redes de valor es la innovación que están aplicando los grandes diarios y grupos audiovisuales globales. La hipótesis planteada se contrasta positivamente a través de los ecosistemas de blogs y software libre para la creación de páginas (page builders) para la autocomunicación de los lectores que está promoviendo Jeff Bezos en The Washington Post, la plataforma de colaboración ciudadana (Taste) de la $B B C$, los proyectos GitHub y Madison de crowdsourcing de The New York times, la plataforma Blendle (iTunes de prensa) de Axel Springer, las iniciativas de crowdfunding como la De Correspondent, la red de WhatsApp de Helsingin Sanomat de Finlandia y su nueva arquitectura de usabilidad publicitaria, los cascos de realidad virtual del Des Moines Register para periodismo inmersivo, las nuevas narrativas transmedia o el uso de inteligencia artificial (automatización y robotización incluida) para determinados procesos y las estrategias de comercialización de cine, videojuegos y televisión de los grupos audiovisuales.

La estructura europea del audiovisual está muy fragmentada y 35 de los 50 principales grupos son americanos, japoneses y coreanos

\section{Nota}

1. Según Manuel Castells, la autocomunicación de masas es "la comunicación que seleccionamos nosotros mismos, pero que tiene el potencial de llegar a masas en términos generales, o a las personas o grupos de personas que seleccionamos en nuestras redes sociales". 
En su libro Comunicación y poder, Castells (2009) detalla: "es comunicación de masas porque potencialmente puede llegar a una audiencia global, como cuando se cuelga un vídeo en YouTube, un blog con enlaces RSS... Al mismo tiempo, es autocomunicación porque uno mismo genera el mensaje, define los posibles receptores y selecciona los mensajes concretos o los contenidos en la web" (pág. 88).

\section{Referencias bibliográficas}

Albarran, Alan B. (1997). Management of electronic media. Bloomington: Wadsworth Publishing Company, Indiana University. ISBN: 0534262740

Albarran, Alan B. (2014). "Assessing the field of media management and economics research: Looking back, looking forward". Palabra clave, v. 17, n. 4, pp. 1025-1040.

http://dx.doi.org/10.5294/pacla.2014.17.4.2

Allee, Verna; Schwabe, Oliver (2011). Value networks and the true nature of collaboration. ValuetNet Works, ed. ISBN: 978615437651

http://www.valuenetworksandcollaboration.com

Benghozi, Pierre-Jean (2006). "Les communautés virtuelles: structuration sociale ou outil de gestion?". Entreprises et histoire, n. 43, pp. 67-81.

https://www.cairn.info/article.php?ID_ARTICLE=EH_043_0067

Benghozi, Pierre-Jean (2011). "Économie numérique et industries de contenu: un nouveau paradigme pour les résaux". Hermes, pp. 31-38. Paris: CNRS-Éditions.

https://halshs.archives-ouvertes.fr/hal-00656840

Bolter, Jay-David; Grusin, Richard (2000). Remediation. Understanding new media. Cambridge: MIT Press, 312 pp. ISBN: 9780262522793

Bourdieu, Pierre (1986). "The forms of capital". En: Richards J. G. (ed), Handbook of theory and research for the sociology of education. New York: Greenwood Publishing Group, pp. 241-258. ISBN: 9780313235290

Bower, Joseph L.; Christensen, Clayton M. (1995). "Disruptive technologies: Catching the wave". Harvard business review, v. 73, n. 1, pp. 43-53. ISSN: 0017-8012

https://hbr.org/1995/01/disruptive-technologies-catchingthe-wave

Bower, Joseph L.; Christensen, Clayton M. (1997). “Disruptive technologies: Catching the wave." En: Seely, Brown, John. Seeing differently: Insights on innovation. Boston, MA: Harvard Business School Press. ISBN: 0875847552

Boyd, Danah M.; Ellison, Nicole B. (2007). "Social network sites: definition, history, and scholarship". Journal of computer-mediated communicaton, v. 13, n. 1, pp. 210-230. http://dx.doi.org/10.1111/j.1083-6101.2007.00393.x

Burt, Ronald S. (1992). Structural holes: The social structure of competition. Cambridge, MA: Harvard University Press. ISBN: 9780674843714

Campos-Freire, Francisco (coord.) (2011). El nuevo escenario mediático. Zamora-Salamanca: Comunicación Social, Colección Periodísctica, n. 44. ISBN: 9788492860630
Castells, Manuel (2009). Comunicación y poder. Madrid: Alianza Editorial, 680 pp. ISBN: 9788420684994

Celaya, Javier et al. (2014). Nuevos modelos de negocio en la era digital. Un estudio elaborado por Dosdoce.com para Cedro con motivo del lanzamiento de la plataforma Conlicencia.com. ISBN: 9788494229589

http://www.dosdoce.com/articulo/estudios/3924/nuevosmodelos-de-negocio-en-la-era-digital

Christensen, Clayton M. (1997). The innovator's dilemma: When new technologies cause great firms to fail. Harvard Business Press. ISSN: 0017-8012

http://www.hbs.edu/faculty/Pages/item.aspx?num =46

Christensen, Clayton M. (2014). “Disruptive innovation”. In: Soegaard, Mads; Dam, Rikke-Friis (eds.). The encyclopedia of human-computer interaction, $2^{\text {nd }}$ ed. Aarhus, Denmark: The Interaction Design Foundation. ISBN: 9788792964007 https://www.interaction-design.org/encyclopedia/ disruptive_innovation.html

Christensen, Clayton M.; Bower, Joseph L. (1995). “Disruptive technologies: Catching the wave". Harvard business review. ISSN: 0017-8012

https://hbr.org/1995/01/disruptive-technologies-catchingthe-wave

Coleman, James S. (1988). "Social capital in the creation of human capital". The American journal of sociology, v. 94, Supplement: Organizations and institutions: Sociological and economic approaches to the analysis of social structure, pp. S95-S120. ISSN: 0002-9602, elSSN: 1537-5390.

http://www.jstor.org/stable/2780243

http://courseweb.lis.illinois.edu/ katewill/for-china/ readings/coleman\%201988\%20social\%20capital.pdf

Damásio, Manuel-José; Henriques, Sara (2011). "The relevance of social capital to the experience of Internet use: contributions to the analysis of the relationship between communities and social networks". International journal of interactive communication systems and technologies. Hershey: IGI Global. Technical Report SITI-TR-11-21. ISSN: 21554218, elSSN:2155-4226

http://copelabs.ulusofona.pt/scicommons/index.php/ attachments/single/258

Davenport, Thomas H.; Beck, John C. (2002). La economía de la atención: el nuevo valor de los negocios. Barcelona: Paidós. ISBN: 9788449312243

Díaz-Nosty, Bernardo (2013). La prensa en el nuevo ecosistema informativo. iQue paren las rotativas! Barcelona: Ariel / Fundación Telefónica, 164 pp. ISBN: 9788408112969 http://goo.gl/hMRYcL

Ellison, Nicole; Boyd, Danah (2013). "Sociality through social network sites". En: The Oxford handbook of internet studies (ed. William H. Dutton). Oxford: Oxford University Press, pp. 151-172. ISBN: 9780199589074

http://www.danah.org/papers/2013/SocialityThruSNSpreprint.pdf

Fine, Charles H. (2000). El nuevo ciclo empresarial. Ventajas competitivas en la era de la velocidad. Barcelona: Paidós, 311 pp. ISBN: 9788449309427 
http://file21.medicbooks.org/22g5ej_el-nuevo-cicloempresarial-ventajas-competitivas-en-la-era-de-la-velocid.pdf

Galán-Ugartemendía, José-Ignacio (2012). "Transmedialidad o narrativa transmedia". Lecciones del Portal de Comunicación. InCom, Universidad Autónoma de Barcelona. http://portalcomunicacion.com/lecciones.asp?aut=81

Goyanes-Martínez, Manuel (2013). “Estrategias y modelos de negocio: Aclaración de conceptos y terminología de la prensa en Internet". Estudios sobre el mensaje periodístico, v. 19, n. 1, pp. 419-431.

http://revistas.ucm.es/index.php/ESMP/article/view/42530 http://dx.doi.org/10.5209/rev_ESMP.2013.v19.n1.42530

Granovetter, Mark S. (1974). Getting a job: a study of contacts and careers. Cambridge: Harvard University Press. ISBN: 9780226305813

Jenkins, Henry (2008). Converge culture: La cultura de la convergencia en los medios de comunicación. Barcelona: Paidós. ISBN: 9788449321535

https://stbngtrrz.files.wordpress.com/2012/10/jenkinshenry-convergence-culture.pdf

Jenkins, Henry (2009). Fans, blogueros y videojuegos: la cultura de la colaboración. Barcelona: Paidós. ISBN: 9788449322587

Jenkins, Henry (2010). Piratas de textos: fans, cultura participativa y televisión. Barcelona: Paidós. ISBN: 9788449324055

Kay, Alan; Goldberg, Adele (1977). "Personal dynamic media". Journal computer, v. 10, n. 3.

http://dx.doi.org/10.1109/C-M.1977.217672

Kinder, Marsha (1991). Playing with power in movies, television, and video games: From muppet babies to teenage mutant ninja turtles, University California Press. ISBN: 978 0520077768

http://goo.gl/VztPFI

Küng, Lucy, ed. (2008). The internet and the mass media. Londres: Sage. ISBN: 9781412947350

Lin, Nan (2001). Social capital: A theory of social structure and action. Cambridge: University Press. ISBN: 9780521521673

Manovich, Lev (2005). El lenguaje de los nuevos medios de comunicación: la imagen en la era digital. Barcelona: Paidós. ISBN: 9788449317699

https://uea1arteycomunicacion.files.wordpress.com/2013/09/ manovich-el-legunaje-de-los-nuevos-medios.pdf

Manovich, Lev (2008). Software takes command. New York: Georgetown University.

http://faculty.georgetown.edu/irvinem/theory/ManovichSoftware-Takes-Command-ebook-2008-excerpt.pdf

Manovich, Lev (2013). Software takes command (international texts in critical media aesthetics). New York: Blomsbury. ISBN: 9781623567453

Mayer-Schönberger, Viktor; Cukier, Kenneth (2013). Big data: A revolution that will transform how we live, work, and think. Boston: An Eamon Dolan Book/Houghton Mifflin Harcourt. ISBN: 9781848547919
Nelson, Ted (1965). "A file structure for the complex, the changing, and the indeterminant" Introducing the terms "hypertext" and "hypermedia", TheNewMediaReader, pp. 133-145.

http://www.composingdigitalmedia.org/digitaliteracy/ docs/11-nelson65-03.pdf

Noguera-Vivo, José-Manuel et al. (2014). Economía de la participación. Madrid: Fundación EOI. ISBN: 9788415061 458

http://repositorio.ucam.edu/jspui/bitstream/10952/1081/1/ Econom\%c3\%ada_Participacion_EOI_2014.pdf

O’Reilly, Tim (2005). Web 2.0. Design patterns and business models for the next generation of software.

http://oreilly.com/web2/archive/what-is-web-20.html

OBS (2013, 2014): Yearbook. Television, cinema, video and on-demand audiovisual services. The pan-European picture. Strasbourg: European Audiovisual Observatory. ISBN: 978 9287180179

http://goo.gl/OR4E7b

OCDE (2010). The OECD innovation strategy: Getting a head start on tomorrow. ISBN: 9789264084704

http://www.oecd-ilibrary.org

Osterwalder, Alexander (2004). The business model ontology - a proposition in a design science approach. Lausanne: University of Lausanne (tesis doctoral).

https://goo.gl/VFIGro

Osterwalder, Alexander; Pigneur, Yves; Tucci, Christopher L. (2005). "Clarifying business models: origins, present, and future of the concept". Communications of the Association for Information Systems, v. 16, Article 1.

http://goo.gl/URMtFa

Picard, Robert G. (2006). "Les médias au risque du management et du marketing". Le temps des médias, n. 6, pp. 165174. ISSN 1764-2507.

http://dx.doi.org/10.3917/tdm.006.0165

Picard, Robert G. (2014). "Twilight or new dawn of journalism. Evidence from the changing news ecosystem". Journalism studies, v. 15, n. 5, pp. 500-510. ISSN: 1461-670X. eISSN: 1469-9699

http://www.robertpicard.net/files/twilight_or_new_dawn_ journalism.pdf

http://dx.doi.org/10.1080/1461670X.2014.895530

Porter, Michael E. (2009). Ser competitivo. Barcelona: Ediciones Deusto. ISBN: 9788423426959

Putnam, Robert D. (1993). "The prosperous community: Social capital and public life". The American prospect, v. 13, pp. 35-42. ISSN: 1049-7285

http://www.prospect.org/article/prosperous-communitysocial-capital-and-public-life

PWC (2014). "Global entertainment and media outlook 2014-2018".

http://www.pwc.com/gx/en/global-entertainment-media-outlook

Requena-Santos, Félix (2003, 2012). Análisis de redes sociales. Orígenes, teorías y aplicaciones. Madrid: CIS. ISBN: 9788474763478 
Rushkoff, Douglas (2014). "The next big think. On the economy of likes", Rhapsody Magazine, September, p. 30.

$h t t p: / / g o o . g l / f S k P b G$

SABI (2014). Base de datos de Análisis de Balances Ibéricos de Empresas.

http://sabi.bvdep.com/ip

Schmidt, Eric; Cohen, Jared (2014). The new digital age. Reshaping the future of people, nations and business. New York: Alfred K. Knopf. ISBN: 9780307957139

Schumpeter, Joseph A. (1943). Capitalism, socialism and democracy. Londres: Routledge. Edic. en español (1984), Barcelona: Ed. Folio.

http://digamo.free.fr/capisoc.pdf

Scolari, Carlos-Alberto (2008). Hipermediaciones. Elementos para una teoría de la comunicación digital interactiva. Barcelona: Gedisa. ISBN: 9788497842730

Sinclair, John (2000). Televisión: comunicación global y regionalización. Barcelona: Gedisa. ISBN: 9788474327854

Stenger, Thomas; Coutant, Alexandre (2011). "Ces réseaux numériques dits sociaux". Hermès, n. 59. Introduction: pp.

\section{9-20. CNRS. ISBN: 9782271071651}

Sullivan, Margareth (2013). "Pledging clarity, The Times plunges into native advertising". The New York times, blog de la Defensora de los lectores del NYT, 19-12-2013. http://publiceditor.blogs.nytimes.com/2013/12/19/ pledging-clarity-the-times-plunges-into-native-advertising

Tapscott, Don; Williams, Anthony D. (2007). Wikinomics: Ia nueva economía de las multitudes inteligentes. Barcelona: Paidós. ISBN: 9788449320149

TNYT (2014). Innovation, The New York Times.

http://www.nytinnovation.com

Vitalari, Nicholas; Shaughnessy, Haydn (2012). The elastic enterprise: The new manifesto for business revolution. Telemachus Press. ISBN: 9781938135347

WAN-IFRA (2014). "World trends database". Asociación Mundial de Diarios, WAN-IFRA.

http://www.wan-ifra.org

WNMN (2014). World News Media Network. ISBN: 9780 989520713

http://www.wnmn.org

\section{INFORMES THINKEPI 2015 SOBRE DOCUMENTACIÓN Y COMUNICACIÓN}

Baiget, Tomàs (dir); Olea, Isabel (coord.) (2015). Informes ThinkEPI 2015. 17x24 cm. Barcelona: Editorial EPI, 213 pp. ISBN: 9788460682097

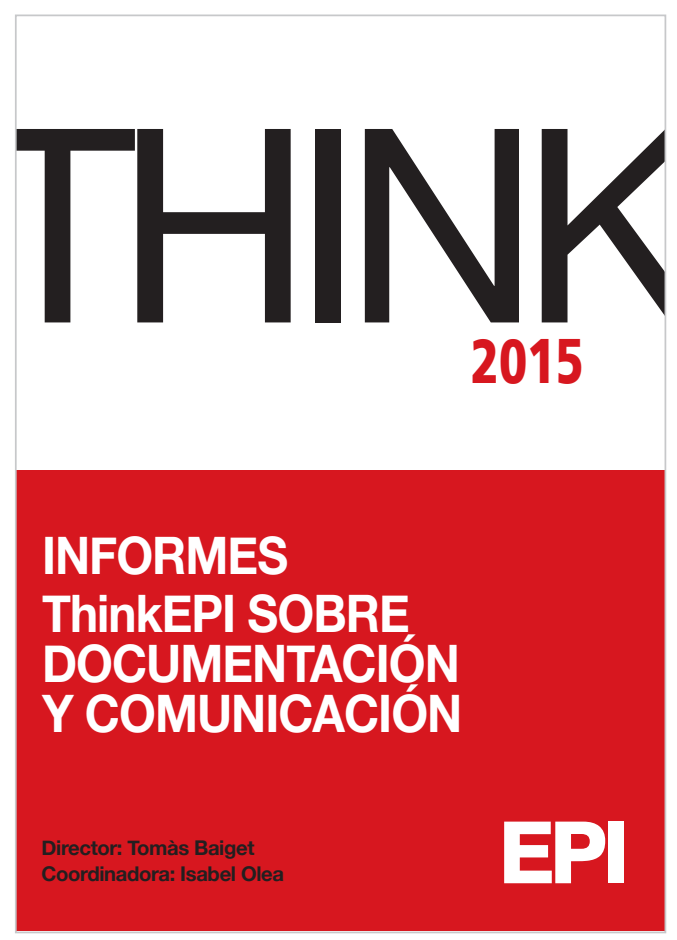

Pedidos: Isabel Olea

epi.iolea@gmail.com
Prólogo: La gran disrupción: la Web + los dispositivos móviles y lo que podemos hacer

\section{Lluís Codina}

Bibliotecas virtuales en 2014, año de consolidación del modelo linked open data

\section{Xavier Agenjo-Bullón}

Tecnología móvil y bibliotecas en 2014: ampliando el concepto de movilidad

\section{Natalia Arroyo-Vázquez}

Encuesta internacional de automatización de bibliotecas Marshall Breeding

Moocs: estado actual, retos y oportunidades

Josep Cobarsí-Morales

Los libros electrónicos: principales tendencias y prospectiva José-Antonio Cordón-García

Servicios de suscripción de libros electrónicos: modelos y tendencias

\section{José-Antonio Cordón-García}

Comunicación científica en 2014. En torno a la 'altmetría' Rodrigo Costas

Informe de la situación de los medios sociales en el sector de la información y la documentación en 2014 y prospectiva 2015 Nieves González-Fernández-Villavicencio

Medios de comunicación digitales en 2014: un balance alternativo

Pere Masip

Acceso abierto en 2014: se sigue avanzando Remedios Melero y Ernest Abadal

Web semántica. Informe de situación 2014

Juan-Antonio Pastor-Sánchez y Tomás Saorín

Resumen de actividades de IFLA, Eblida, Liber y Fesabid en 2014

Glòria Pérez-Salmerón

Informe de situación 2015: profesión y formación universitaria Carlos-Miguel Tejada-Artigas 